\title{
Daily Fluctuation in Solid Waste Generation in Rural Areas of Jammu (A Case Study In Parwah Village, Jammu)
}

\author{
Deepika Sharma and Raj Kumar Rampal \\ Department of Environmental Sciences University of Jammu
}

\begin{abstract}
To plan a municipal solid waste management strategies for a given region, it is essential to know the quantity of waste generated. Past research have shown that amount of waste generated is proportional to the population and the average income of the people. This paper presents the findings of the study carried out in rural area at Parwah village of Block Marh, Jammu to determine solid waste generation rate on income bases. Average solid waste generation/week, /month and per year of study area was calculated. Co-relation coefficient (r) between no. of individuals (human and animals) and solid waste generation /house/day in high, middle and low income households were also calculated and the results of the present study revealed that wealthier strata produces higher waste generation followed by low and middle income respectively. Overall compilation of the data of all the households of the study area reveal that the average solid waste generation in average household of the study area was observed to be $6561.543 \pm 1837.35 \mathrm{~g} / \mathrm{house} /$ day i.e. $6.561 \pm 1.837 \mathrm{~kg} / \mathrm{house} /$ day. The generation of solid waste was observed to be positively correlated with family size in all types of households i.e. high income households $(r=0.77, p<0.05)$, middle income households $(r=0.59, p<0.05)$ and low income households ( $r=0.1, p<0.05)$.

Keywords: Waste, Households, Income, Rural.
\end{abstract}

\section{Introduction}

Solid waste is defined as unwanted or discarded waste material from houses, institutions, hospitals, extraction waste and energy production. Solid waste predominantly includes food waste, containers and product packaging and other miscellaneous inorganic waste from residential, commercial, institutional and industrial sources. Solid waste problems which we are facing today have their roots since the beginning of human kind, when man slaughtered animals for food or cut the wood to make their carts. With progress of the civilization, the waste generation became more complex in nature and the end of $19^{\text {th }}$ century witnessed a rise of world of consumers thereby generating more of non-biodegradable waste as compared to bio-degradable due to urbanization and industrialization .Excessive and increasing land pollution is endangering life on the earth and harmful solid waste have changed the earth's natural balance. Poor solid waste management resulted into two major problems, depletion of resources and increase in pollution loads.

A lot of work has been done on solid waste generation in urban areas by a number of workers like Mukherjee and Roy (1989) in Calcutta, Sinha and Rawat (1991) in Jaipur, Chakarabarti (1993) in Hyderabad, Pasrija et al. (1995) in Chandigarh, Rao and Shantaram (1996) in Hyderabad, Sharholy et al. (2007) in Indian cities, Gamse and Medina (2010) in Indonesia, Ansari (2012) in Bahrain district.

But few workers have done on solid waste generation in rural areas. In present investigation study has been carried out to assess the daily variation in solid waste generation in the households of the village Parwah which is located in outskirts of Jammu city, about $12 \mathrm{~km}$ from Jammu in westward direction. It does not lie under municipal limits. Majority of people in this village fall under middle income category and depend on agriculture for their livelihood, as the area possesses very fertile soil. This area is comprised of about 250 houses and 1100 people, 26 shops and 7 educational institutes.

\section{Methodology}

Sampling of solid waste were done randomly in 30 households of the village Parwah selecting 10 households of high income (with monthly income >20,000), 10 households of middle income (with monthly income 10,000-20,000) and 10 households of low income category (with monthly income <10,000). At each household sampling of solid waste was done thrice for all the days of a week. During each sampling solid waste generated during $24 \mathrm{hrs}$ a day was collected and segregated into bio-degradable solid waste/house/day and nonbiodegradable solid waste/house/day by weighing on a digital balance. During the sampling period no. of family members as well as number of cattles were also recorded to calculate average biodegradable waste/capita/day, average non-biodegradable solid waste/capita/day and average total solid waste generated in $\mathrm{gm} / \mathrm{capita} / \mathrm{house} / \mathrm{day}$. All the average values of solid waste were further compiled to calculate average solid waste generation per high income household, middle income household and low income household and finally average solid waste generation per household of study area. Average solid waste generation per week, per 
month and per year of study area was also calculated. Correlation coefficient (r) between number of individuals ( human and animal) and Solid waste generation /house/day in high income household, middle income household and low income household were also calculated using Pearson product moment method.

\section{Observation and Discussion (Tables I - V)}

Overall critical analysis of all the three categories of households of the study area reveal that high income households of the study area exhibited higher value of solid waste generation followed by low income and least value is exhibited by middle income households. Qdais et al. 1997 reported that solid waste generation was dependent on the income level. From this work it can be concluded that high income households generate more solid waste than low income households and this is in support of the observation made in the present study. The generation of solid waste was observed to be positively correlated with family size in all types of households i.e. high income households $(r=0.77, p<0.05)$, middle income households $(r=0.59, p<0.05)$ and low income households $(\mathrm{r}=0.1, \mathrm{p}<0.05$ ). Sujauddin et al 2008 while investigating per capita waste generation by residents, its composition, and the households' attitudes towards waste management at Rahman Nagar Residential Area, Chittagong, Bangladesh also reported that the generation of household solid waste was positively correlated with family size $\left(r_{x y}=0.236, p<0.05\right)$, education level $\left(r_{x y}=0.244, p<0.05\right)$ and monthly income $\left(r_{x y}=0.671, p<0.01\right)$ of the households.

Overall compilation of the data of all the households of the study area reveal that the average solid waste generation in average household of the study area was observed to be $6561.543 \pm 1837.35 \mathrm{~g} / \mathrm{house} /$ day i.e.6.561 $\pm 1.837 \mathrm{~kg} /$ house/day. Since the approximate no. of households in the study area was observed to be 250 so the average solid waste generation in the study area was calculated to be $1640.38 \mathrm{~kg} / \mathrm{day}$ comprising $1631.17 \mathrm{~kg} /$ day biodegradable waste and $9.20 \mathrm{~kg} / \mathrm{day}$ of non-biodegradable solid waste. .Overall compilation of the data the of average solid waste generation in the study area on weekly, monthly and yearly basis revealed the values of $11482.66 \mathrm{~kg} /$ week, $49211.4 \mathrm{~kg} / \mathrm{month}$ and $598738.7 \mathrm{~kg} /$ year respectively.

\section{Management}

The observation on present scenario of solid waste management revealed that the animal waste is used as i) fertilizer in the agricultural fields, ii) used to make cow dung cakes used as fuel, iii) used to plaster walls and floors. Other bio-degradable as well as non-bio-degradable solid waste is disposed off in open drains, in the nearby canals after packing the waste in the polythene bags, and used to burn the solid waste to get rid of it. Some leftover of the kitchen is also be used as feed for animals. Since the area is not under municipal limits, so no worker of the municipal committee use to dispose off solid waste and all the solid waste disposal is done by the local residents in both eco-friendly and unethical manner. Some of the bio-degradable and nonbiodegradable solid waste is also picked up by the rag pickers during their frequent visits to the study area. Due to lack of proper management of the solid waste the solid waste in the dumping places become breeding grounds for the germs and attractive places for the stray animals and some cattle like cow buffalo etc.

It is an old saying "cleanliness is next to Godliness" and cleanliness is an indicator of good rural management. From the above observations the following recommendations can be made to reduce problems due to solid waste:

The collection of waste by municipal committee through vehicle should be done regularly so that waste does not remain scattered on the roads. People should be properly aware to dispose off waste in an eco-friendly and hygienic manner. Government and NGO's should educate people regarding the solid waste management. Segregation and separation of reusable and recyclable materials should be encouraged. People should be educated regarding waste reuse, recycling and reclamation. They must not let animal waste of agricultural runoff to enter in any water body which can enhance eutrophication 
AVERAGE SOLID WASTE GENERATED IN HIGH INCOME HOUSEHOLDS DURING DIFFERENT DAYS OF A WEEK (Table I)

\begin{tabular}{|c|c|c|c|c|c|c|c|c|c|c|}
\hline \multirow{3}{*}{ DAY. } & \multicolumn{5}{|c|}{ Solid waste generation (gm)/house/day } & \multicolumn{5}{|c|}{ Solid waste generation (gm)/capita/day } \\
\hline & \multicolumn{3}{|c|}{ BSW } & \multirow[t]{2}{*}{ NBSW } & \multirow[t]{2}{*}{ Total } & \multicolumn{3}{|c|}{ BSW } & \multirow[t]{2}{*}{ NBSW } & \multirow[t]{2}{*}{ Total } \\
\hline & Domestic & $\begin{array}{l}\text { Animal } \\
\text { shed }\end{array}$ & Total & & & $\begin{array}{l}\text { Domes } \\
\text { tic }\end{array}$ & $\begin{array}{l}\text { Animal } \\
\text { shed }\end{array}$ & Total & & \\
\hline$\underline{\text { SUN }}$ & $517.2 \pm$ & $8520 \pm$ & $9037.2 \pm$ & $67.9 \pm$ & $9105.1 \pm$ & $95.53 \pm$ & $2600 \pm$ & $2695.53 \pm$ & $14.47 \pm$ & $2710.0 \pm$ \\
\hline$\underline{D A Y}$ & $\begin{array}{l}166.48 \\
(402-910)\end{array}$ & $\begin{array}{l}13205.79 \\
(0- \\
39000)\end{array}$ & $\begin{array}{l}13309.25 \\
(402- \\
39910)\end{array}$ & $\begin{array}{l}31.51 \\
(34- \\
120)\end{array}$ & $\begin{array}{l}13300.19 \\
(484- \\
39949)\end{array}$ & $\begin{array}{l}11.84( \\
74.83- \\
108)\end{array}$ & $\begin{array}{l}3285.997( \\
0-7800)\end{array}$ & $\begin{array}{l}3283.58 \\
(79.5- \\
7891)\end{array}$ & $\begin{array}{l}9.54 \\
(3.9- \\
29.25)\end{array}$ & $\begin{array}{l}3282.83 \\
(87.66- \\
7894.9)\end{array}$ \\
\hline MON & $472.1 \pm$ & $8130 \pm$ & 8602.11 & $76.6 \pm$ & $8678.7 \pm 130$ & 86.497 & $2447.5 \pm$ & $2533.99 \pm$ & $12.17 \pm$ & $2546.17 \pm$ \\
\hline$\underline{D A Y}$ & $\begin{array}{l}174.64 \\
(342-869)\end{array}$ & $\begin{array}{l}12880.74 \\
(0- \\
38500)\end{array}$ & $\begin{array}{l} \pm 2990.70 \\
(350- \\
39369)\end{array}$ & $\begin{array}{l}107.19 \\
(8-295)\end{array}$ & $\begin{array}{l}89.57 \\
(380- \\
39664)\end{array}$ & $\begin{array}{l} \pm \\
13.31 \\
(66.83- \\
100.71\end{array}$ & $\begin{array}{l}2974.205 \\
(0-7700)\end{array}$ & $\begin{array}{l}2971.96 \\
(66.83- \\
1800.71)\end{array}$ & $\begin{array}{l}13.77 \\
(2- \\
43.33)\end{array}$ & $\begin{array}{l}2981.12 \\
(68.49- \\
1802.71)\end{array}$ \\
\hline TUES & $492.6 \pm$ & $4600 \pm 69$ & $5092.6 \pm$ & $28 \pm$ & $5120.6 \pm 697$ & $90.96 \pm$ & $2405 \pm 302$ & $2495.96 \pm$ & $5.31 \pm$ & $2501.28 \pm$ \\
\hline$\underline{D A Y}$ & $\begin{array}{l}171.32 \\
(360-900)\end{array}$ & $\begin{array}{l}99.841 \\
(0- \\
20000)\end{array}$ & $\begin{array}{l}6969.95 \\
(380- \\
20411)\end{array}$ & $\begin{array}{l}10.28 \\
(10-42)\end{array}$ & $\begin{array}{l}5.139(390- \\
20453)\end{array}$ & $\begin{array}{l}15.24 \\
(68.5- \\
112.25 \\
)\end{array}$ & $\begin{array}{l}5.673(0- \\
7400)\end{array}$ & $\begin{array}{l}3021.87 \\
(71.16- \\
7490)\end{array}$ & $\begin{array}{l}2.024 \\
(2.5- \\
8.75)\end{array}$ & $\begin{array}{l}3022.16 \\
(74.32- \\
7494.1)\end{array}$ \\
\hline WEDN & $485.5 \pm$ & $8500 \pm 13$ & $8985.5 \pm$ & $20.8 \pm$ & $9006.3 \pm 133$ & $89.64 \pm$ & $\mathbf{2 5 7 5 . 5} \pm 3$ & $2665.14 \pm$ & $3.88 \pm$ & $2669.029 \pm 3$ \\
\hline$\frac{\underline{E S D A}}{\underline{Y}}$ & $\begin{array}{l}168.49 \\
(302-876)\end{array}$ & $\begin{array}{l}294.94 \\
(0-\end{array}$ & $\begin{array}{l}13388.79 \\
(412-\end{array}$ & $\begin{array}{l}7.42 \\
(10-31)\end{array}$ & $\begin{array}{l}91.93 \\
(422-\end{array}$ & $\begin{array}{l}15.49 \\
(60.4\end{array}$ & $\begin{array}{l}257.479 \\
(1200-\end{array}$ & $\begin{array}{l}3251.52 \\
(79.5-\end{array}$ & $\begin{array}{l}1.22 \\
(2.5-5.5)\end{array}$ & $\begin{array}{l}251.099 \\
(84.5-\end{array}$ \\
\hline & & $39400)$ & 40276) & & 40305) & )$^{105.25}$ & $7880)$ & 7967.6) & & $7970.5)$ \\
\hline THUR & $482.6 \pm$ & $8750 \pm 13$ & $9232.6 \pm$ & $29.4 \pm$ & $9262 \pm$ & $88.44 \pm$ & $2755 \pm 327$ & $2843.44 \pm$ & $5.54 \pm$ & $2848.98 \pm$ \\
\hline SDAY & $\begin{array}{l}187.32 \\
(282-892)\end{array}$ & $\begin{array}{l}305.16 \\
(0- \\
39500)\end{array}$ & $\begin{array}{l}13416.79 \\
(282- \\
40392)\end{array}$ & $\begin{array}{l}7.76 \\
(19-38)\end{array}$ & $\begin{array}{l}13421.35 \\
(302- \\
40434)\end{array}$ & $\begin{array}{l}18.95 \\
(57.8- \\
115.5)\end{array}$ & $\begin{array}{l}9.096(0- \\
7900)\end{array}$ & $\begin{array}{l}3278.12 \\
(70.5- \\
7989.2)\end{array}$ & $\begin{array}{l}1.30 \\
(4-8)\end{array}$ & $\begin{array}{l}3278.30 \\
(75.5- \\
7993.4)\end{array}$ \\
\hline FRI & $591.2 \pm$ & $8722 \pm 13$ & $9313.2 \pm$ & $50.2 \pm$ & $9363.4 \pm$ & 109.61 & $2662.5 \pm 3$ & $2772.11 \pm$ & $9.92 \pm$ & $2782.03 \pm$ \\
\hline$\underline{\mathrm{DAY}}$ & $\begin{array}{l}196.37 \\
(462- \\
1100)\end{array}$ & $\begin{array}{l}571.14(0 \\
-40200)\end{array}$ & $\begin{array}{l}13710.68 \\
(480- \\
41300)\end{array}$ & $\begin{array}{l}14.92 \\
(30-82)\end{array}$ & $\begin{array}{l}13718.13 \\
(526- \\
41355)\end{array}$ & $\begin{array}{l} \pm \\
18.12 \\
(86.83- \\
145.5)\end{array}$ & $\begin{array}{l}323.189 \\
(0-8040)\end{array}$ & $\begin{array}{l}3319.18 \\
(100.2- \\
8150)\end{array}$ & $\begin{array}{l}3.97 \\
(5.5- \\
15.5)\end{array}$ & $\begin{array}{l}3319.63 \\
(93.5- \\
8155.5)\end{array}$ \\
\hline$\underline{\text { SATU }}$ & $474.1 \pm$ & 8740 & $9214.1 \pm$ & $43 \pm$ & $9257.1 \pm$ & 88.114 & $2722 \pm 332$ & $2810.11 \pm$ & $8.110 \pm$ & $2818.224 \pm$ \\
\hline$\underline{\mathrm{RDAY}}$ & $\begin{array}{l}152.34 \\
(324-802)\end{array}$ & $\begin{array}{l} \pm 13441.8 \\
3 \quad(0- \\
40100)\end{array}$ & $\begin{array}{l}13520.39 \\
(347- \\
40902)\end{array}$ & $\begin{array}{l}15.28 \\
(9-63)\end{array}$ & $\begin{array}{l}13527.60 \\
(378- \\
40953)\end{array}$ & $\begin{array}{l} \pm \\
17.017 \\
(64.8- \\
120)\end{array}$ & $\begin{array}{l}7.037(0- \\
8020)\end{array}$ & $\begin{array}{l}3321.38 \\
(76.84- \\
8100.2)\end{array}$ & $\begin{array}{l}3.062 \\
(2.25- \\
12.6)\end{array}$ & $\begin{array}{l}3322.281 \\
(83.24- \\
8105.3)\end{array}$ \\
\hline$\frac{\text { Avg./da }}{\text { y }}$ & $\begin{array}{l}\mathbf{5 0 2 . 1 8 5 7} \\
\pm \\
\mathbf{4 2 . 0 2 ( 4 7} \\
2.1- \\
591.2-)\end{array}$ & $\begin{array}{l}7994.571 \\
\pm \\
1512.64 \\
(4600- \\
8750)\end{array}$ & $\begin{array}{l}\mathbf{8 4 9 6 . 7 5 \pm} \\
\mathbf{1 5 1 9 . 4 8} \\
(5092.6- \\
9313.2)\end{array}$ & $\begin{array}{l}\mathbf{4 5 . 1 2 \pm} \\
\mathbf{2 1 . 1 0} \\
(20.8- \\
76.6)\end{array}$ & $\begin{array}{l}\mathbf{8 5 4 1 . 8 8} \pm \mathbf{1 5} \\
\mathbf{2 5 . 4 3 9} \\
(5120.6- \\
9363.4-)\end{array}$ & $\begin{array}{l}92.68 \pm \\
8.00 \\
(86.49- \\
109.61 \\
)\end{array}$ & $\begin{array}{l}\mathbf{2 5 9 5 . 3 5 7} \\
\pm \\
\mathbf{1 3 2 . 0 0 ( 2 4} \\
05-2755)\end{array}$ & $\begin{array}{l}\mathbf{2 6 8 8 . 0 4 \pm} \\
\mathbf{1 3 3 . 7 4} \\
(2495.96- \\
2843.44)\end{array}$ & $\begin{array}{l}8.48 \pm \\
3.90 \\
(3.88- \\
14.47)\end{array}$ & $\begin{array}{l}\mathbf{2 6 9 6 . 5 3} \pm \mathbf{1 3} \\
\mathbf{3 . 5 5 9 7} \\
(2501.28- \\
2848.98)\end{array}$ \\
\hline
\end{tabular}


AVERAGE SOLID WASTE GENERATED IN MIDDLE INCOME HOUSEHOLDS DURING DIFFERENT DAYS OF A WEEK (Table II)

\begin{tabular}{|c|c|c|c|c|c|c|c|c|c|c|}
\hline \multirow{3}{*}{$\begin{array}{l}\text { DA } \\
\mathrm{Y} .\end{array}$} & \multicolumn{5}{|c|}{ Solid waste generation (gm)/house/day } & \multicolumn{5}{|c|}{ Solid waste generation (gm) /capita/day } \\
\hline & \multicolumn{3}{|l|}{ BSW } & \multirow{2}{*}{$\begin{array}{l}\text { NBS } \\
\mathrm{W}\end{array}$} & \multirow[t]{2}{*}{ Total } & \multicolumn{3}{|c|}{ BSW } & \multirow[t]{2}{*}{ NBSW } & \multirow[t]{2}{*}{ Total } \\
\hline & $\begin{array}{l}\text { Domesti } \\
\mathrm{c}\end{array}$ & $\begin{array}{l}\text { Animal } \\
\text { shed }\end{array}$ & Total & & & $\begin{array}{l}\text { Domesti } \\
\mathrm{c}\end{array}$ & $\begin{array}{l}\text { Animal } \\
\text { shed }\end{array}$ & total & & \\
\hline $\begin{array}{l}\frac{\mathbf{S U}}{\mathbf{N}} \\
\frac{\overline{\mathrm{D} A}}{\underline{Y}}\end{array}$ & $\begin{array}{l}\mathbf{3 1 9 . 1} \pm \\
50.08 \\
(260- \\
397)\end{array}$ & $\begin{array}{l}\mathbf{4 9 0 0} \pm 5 \\
489.789 \\
(0- \\
12100)\end{array}$ & $\begin{array}{l}\mathbf{5 2 1 9 . 1 \pm} \\
5476.23 \\
(262- \\
12360)\end{array}$ & $\begin{array}{l}\mathbf{3 1 . 4} \pm \\
18.08 \\
(9-64)\end{array}$ & $\begin{array}{l}\mathbf{5 2 5 0 . 5} \\
\pm \\
5487.09 \\
4(290- \\
12424) \\
\end{array}$ & $\begin{array}{l}\text { 71.12 } \pm \\
16.136 \\
(52.4- \\
99.25)\end{array}$ & $\begin{array}{l}\mathbf{2 4 2 2 . 5} \\
\pm 2679 . \\
823(0- \\
6050)\end{array}$ & $\begin{array}{l}\text { 2493.62 } \\
2672.28 \\
(52.4- \\
6115)\end{array}$ & $\begin{array}{l}\mathbf{6 . 9 4} \pm \\
4.234 \\
(2.25- \\
10.4)\end{array}$ & $\begin{array}{l}\mathbf{2 5 0 0 . 5 6} \\
\pm \\
2674.03 \\
(62.8- \\
6131) \\
\end{array}$ \\
\hline $\begin{array}{l}\frac{\text { MO }}{\mathbf{N}} \\
\frac{\overline{\mathrm{D}} \mathbf{A}}{\underline{\mathbf{Y}}}\end{array}$ & $\begin{array}{l}\text { 324.3 } \pm \\
33.159 \\
(281- \\
391)\end{array}$ & $\begin{array}{l}\mathbf{4 4 0 0} \pm 5 \\
605.949 \\
(0- \\
12100)\end{array}$ & $\begin{array}{l}\mathbf{4 7 2 4 . 3 \pm} \\
5599.67 \\
(296- \\
12381)\end{array}$ & $\begin{array}{l}\mathbf{2 1 . 3} \\
\pm \\
13.33 \\
(9-47)\end{array}$ & $\begin{array}{l}\mathbf{4 7 4 5 . 6} \\
\pm \\
5601.35 \\
(308- \\
12392)\end{array}$ & $\begin{array}{l}\mathbf{7 1 . 8 2} \pm \\
10.132 \\
(55.16- \\
86)\end{array}$ & $\begin{array}{l}\mathbf{1 9 4 5} \pm \\
2537.4 \\
36(0- \\
6050)\end{array}$ & $\begin{array}{l}\text { 2016.82 } \\
2534.35 \\
(74- \\
6120.25)\end{array}$ & $\begin{array}{l}\mathbf{4 . 7 5} \pm \\
3.068 \\
(1.8- \\
11)\end{array}$ & $\begin{array}{l}\mathbf{2 0 2 1 . 5 7} \\
\pm 2535.4 \\
57 \\
(61.6- \\
6123)\end{array}$ \\
\hline$\frac{\frac{\mathrm{TU}}{\mathrm{ES}}}{\frac{\underline{\mathrm{DA}}}{\underline{Y}}}$ & $\begin{array}{l}\mathbf{3 5 4 . 6 \pm} \\
50.089 \\
(288- \\
422)\end{array}$ & $\begin{array}{l}\mathbf{4 6 3 0} \pm 5 \\
251.254 \\
(0- \\
12700)\end{array}$ & $\begin{array}{l}\mathbf{4 9 8 4 . 6 \pm} \\
5240.62 \\
(307- \\
12990)\end{array}$ & $\begin{array}{l}\mathbf{2 4 . 5} \pm \\
13.30 \\
(7-51)\end{array}$ & $\begin{array}{l}\mathbf{5 0 0 9 . 1 \pm} \\
5250.23 \\
(321- \\
13021)\end{array}$ & $\begin{array}{l}\mathbf{7 9 . 4 7 \pm} \\
18.38 \\
(48- \\
105.5)\end{array}$ & $\begin{array}{l}\mathbf{2 0 4 5} \pm \\
2648.6 \\
32(0- \\
6350)\end{array}$ & $\begin{array}{l}\mathbf{2 1 2 4 . 4 7 \pm} \\
2641.49 \\
(64.8- \\
6422.5)\end{array}$ & $\begin{array}{l}\mathbf{5 . 4 1 \pm} \\
2.985 \\
(1.75- \\
10.2)\end{array}$ & $\begin{array}{l}\mathbf{2 1 2 9 . 8 8} \\
\pm \\
2642.05 \\
5 \\
(69.2- \\
6430.25 \\
)\end{array}$ \\
\hline$\frac{\frac{W E}{D N}}{\frac{E S}{D A}}$ & 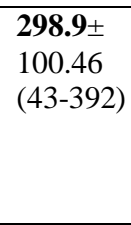 & $\begin{array}{l}3820 \pm 5 \\
314.50( \\
0- \\
12900)\end{array}$ & 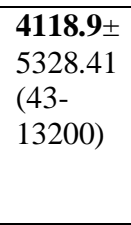 & $\begin{array}{l}\mathbf{2 7 . 2 \pm} \\
8.21 \\
(14- \\
42)\end{array}$ & $\begin{array}{l}\text { 4146.1 } \pm \\
5333.12 \\
(57- \\
13229)\end{array}$ & $\begin{array}{l}\mathbf{6 6 . 0 6} \pm \\
24.68 \\
(10.75- \\
95.25)\end{array}$ & $\begin{array}{l}\mathbf{1 8 7 7 . 5} \\
\pm 2575 \\
214(0- \\
6450)\end{array}$ & $\begin{array}{l}\text { 1943.56 } \pm \\
2571.52 \\
(10.75- \\
6525)\end{array}$ & $\begin{array}{l}\mathbf{5 . 9 5 1 \pm} \\
1.70 \\
(3.5- \\
8.5)\end{array}$ & $\begin{array}{l}\text { 1949.51 } \\
\pm \\
2571.72 \\
(14.25- \\
6532.25 \\
)\end{array}$ \\
\hline $\begin{array}{l}\frac{\mathrm{TH}}{\mathrm{UR}} \\
\underline{\mathrm{SD}} \\
\underline{\mathrm{AY}}\end{array}$ & $\begin{array}{l}\text { 433.2 } \\
51.79 \\
(369- \\
501)\end{array}$ & $\begin{array}{l}\mathbf{4 5 0 1} \pm 5 \\
202.041 \\
(0- \\
12200)\end{array}$ & $\begin{array}{l}\text { 4934.2 } \\
5210.62 \\
(402- \\
12580)\end{array}$ & $\begin{array}{l}\mathbf{3 5 . 8 5} \\
\pm \\
11.05 \\
5 \\
(24- \\
62) \\
\end{array}$ & $\begin{array}{l}\mathbf{4 9 7 0 . 0 5} \\
\pm 5218.3 \\
39 \\
(405- \\
12619)\end{array}$ & $\begin{array}{l}96.33 \pm \\
16.95 \\
(63.5- \\
123)\end{array}$ & $\begin{array}{l}\mathbf{2 2 1 3} \pm \\
2499.6 \\
32(0- \\
6100)\end{array}$ & $\begin{array}{l}\mathbf{2 3 0 9 . 3 3 5} \\
\pm \\
2493.724 \\
(84.6- \\
6195)\end{array}$ & $\begin{array}{l}\mathbf{7 . 9 1 8} \pm \\
2.349 \\
(5- \\
12.4)\end{array}$ & $\begin{array}{l}\mathbf{2 3 1 7 . 2 5} \\
\pm \\
2494.29 \\
(89.6- \\
6204.75 \\
) \\
\end{array}$ \\
\hline$\frac{\frac{\text { FRI }}{\mathrm{DA}}}{\underline{\mathrm{Y}}}$ & $\begin{array}{l}\text { 319.4士 } \\
131.32 \\
(71-509)\end{array}$ & $\begin{array}{l}\mathbf{4 8 6 0} \pm 5 \\
445.732 \\
(0- \\
12900)\end{array}$ & $\begin{array}{l}\mathbf{5 1 7 9 . 4 \pm} \\
5470.11 \\
(71- \\
13070)\end{array}$ & $\begin{array}{l}\mathbf{3 8 . 7 \pm} \\
13.87 \\
(20- \\
71)\end{array}$ & $\begin{array}{l}\mathbf{5 2 1 8 . 1 \pm} \\
5476.79 \\
(107- \\
13103)\end{array}$ & $\begin{array}{l}\mathbf{6 8 . 9 4} \pm \\
25.55 \\
(17.75- \\
94.2)\end{array}$ & $\begin{array}{l}\mathbf{2 4 2 5} \pm \\
2710.8 \\
0(0- \\
6450)\end{array}$ & $\begin{array}{l}\text { 2493.94 } \pm \\
2711.10 \\
(65- \\
6492.5)\end{array}$ & $\begin{array}{l}\mathbf{8 . 5 5 4} \pm \\
2.880 \\
(4- \\
14.2)\end{array}$ & $\begin{array}{l}\mathbf{2 5 0 2 . 5 0} \\
\pm \\
2711.35 \\
(26.75- \\
6500.75 \\
)\end{array}$ \\
\hline $\begin{array}{l}\frac{\mathrm{SA}}{\mathrm{TU}} \\
\frac{\mathrm{RD}}{\mathrm{AY}} \\
\underline{y}\end{array}$ & $\begin{array}{l}\text { 353.6 } \pm \\
64.42 \\
(274- \\
472)\end{array}$ & $\begin{array}{l}\mathbf{4 6 4 0} \pm 5 \\
220.515 \\
(0- \\
12000)\end{array}$ & $\begin{array}{l}\text { 4993.6 } \pm \\
5206.43 \\
(314- \\
12274)\end{array}$ & $\begin{array}{l}\mathbf{5 2 . 1 \pm} \\
29.13 \\
(11- \\
89)\end{array}$ & $\begin{array}{l}\mathbf{5 0 4 5 . 7 \pm} \\
5218.00 \\
(339- \\
12350)\end{array}$ & $\begin{array}{l}\mathbf{7 7 . 9 5} \pm \\
13.70 \\
(51.67- \\
107.75)\end{array}$ & $\begin{array}{l}\mathbf{2 0 7 7 . 5} \\
\pm 2439 \\
901 \\
(0- \\
6000)\end{array}$ & $\begin{array}{l}\mathbf{2 1 5 5 . 4 5} \pm \\
2431.90 \\
(77.8- \\
6068.5)\end{array}$ & $\begin{array}{l}\mathbf{1 1 . 3 8} \pm \\
5.96 \\
(2.2- \\
19)\end{array}$ & $\begin{array}{l}\mathbf{2 1 6 6 . 8 3} \\
\pm \\
2434.94 \\
(80- \\
4766.27 \\
)\end{array}$ \\
\hline$\frac{\mathrm{Avg}}{\frac{. / \mathrm{da}}{\mathrm{y}}}$ & $\begin{array}{l}\text { 343.3 } \\
\mathbf{4 4 . 3 5} \\
(298.9- \\
433.2)\end{array}$ & $\begin{array}{l}\mathbf{4 5 3 5 . 8 5} \\
\mathbf{7 \pm} \\
\mathbf{3 6 2 . 7 4 4} \\
\mathbf{4}(3820- \\
4900)\end{array}$ & $\begin{array}{l}\mathbf{4 8 7 9 . 1 5} \\
\mathbf{\pm} \\
\mathbf{3 7 3 . 0 3} \\
(4118.9- \\
5219.1)\end{array}$ & $\begin{array}{l}\mathbf{3 3 . 0 0} \\
\mathbf{\pm} \\
\mathbf{1 0 . 4 1} \\
(21.3- \\
52.1)\end{array}$ & $\begin{array}{l}\mathbf{4 9 1 2 . 1 6} \\
\mathbf{\pm} \\
\mathbf{3 7 7 . 1 0 4} \\
\mathbf{1} \\
(4146.1 \\
- \\
5250.5)\end{array}$ & $\begin{array}{l}\mathbf{7 5 . 9 5} \pm \\
\mathbf{1 0 . 1 5} \\
(66.06- \\
96.33)\end{array}$ & $\begin{array}{l}\mathbf{2 1 4 3 . 6} \\
\mathbf{4 3} \pm 21 \\
\mathbf{8 . 3 7 6 5} \\
(1877 . \\
5- \\
2425)\end{array}$ & $\begin{array}{l}2245.94 \pm \\
\mathbf{2 4 1 . 9 0} \\
(1943.56 \\
- \\
2493.94)\end{array}$ & $\begin{array}{l}\mathbf{7 . 2 7} \pm \\
\mathbf{2 . 2 5} \\
(4.75- \\
11.38)\end{array}$ & $\begin{array}{l}\mathbf{2 2 2 6 . 8 7} \\
\mathbf{1} \pm \mathbf{2 2 0 . 2} \\
\mathbf{7 8}(1949 \\
.51- \\
2502.50 \\
)\end{array}$ \\
\hline
\end{tabular}


AVERAGE SOLID WASTE GENERATED IN LOW INCOME HOUSEHOLDS DURING DIFFERENT DAYS OF A WEEK (Table III)

\begin{tabular}{|c|c|c|c|c|c|c|c|c|c|c|}
\hline \multirow[t]{3}{*}{ DAY. } & \multicolumn{5}{|c|}{ Solid waste generation (gm)/house/day } & \multicolumn{5}{|c|}{ Solid waste generation (gm)/capita/day } \\
\hline & \multicolumn{3}{|l|}{ BSW } & \multirow{2}{*}{$\begin{array}{l}\text { NBS } \\
\text { W }\end{array}$} & \multirow[t]{2}{*}{ Total } & \multicolumn{3}{|l|}{ BSW } & \multirow{2}{*}{$\begin{array}{l}\text { NBS } \\
\mathrm{W}\end{array}$} & \multirow[t]{2}{*}{ Total } \\
\hline & $\begin{array}{l}\text { Domest } \\
\text { ic }\end{array}$ & $\begin{array}{l}\text { Animal } \\
\text { shed }\end{array}$ & Total & & & $\begin{array}{l}\text { Domesti } \\
\mathrm{c}\end{array}$ & $\begin{array}{l}\text { Anim } \\
\text { al } \\
\text { shed } \\
\end{array}$ & Total & & \\
\hline $\begin{array}{l}\text { SUN } \\
\text { DAY }\end{array}$ & 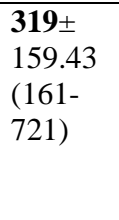 & $\begin{array}{l}\mathbf{5 7 2 1} \pm 5 \\
909.368 \\
(0- \\
16400)\end{array}$ & $\begin{array}{l}\mathbf{6 0 4 0 \pm} \\
5897.04 \\
(198- \\
16742)\end{array}$ & $\begin{array}{l}74.4 \\
\pm \\
33.6 \\
4 \\
(6- \\
111) \\
\end{array}$ & $\begin{array}{l}\mathbf{6 1 1 4 . 4 \pm} \\
5886.61 \\
(277- \\
16748)\end{array}$ & $\begin{array}{l}\mathbf{6 6 . 1 1} \pm 3 \\
9.60 \\
(33-171)\end{array}$ & $\begin{array}{l}\mathbf{2 8 5 0 .} \\
\mathbf{5} \pm 270 \\
1.8 \\
(0- \\
8200)\end{array}$ & $\begin{array}{l}\mathbf{2 9 1 6 . 6 1} \\
\pm \\
2731.54 \\
(33- \\
8371)\end{array}$ & $\begin{array}{l}\mathbf{1 4 . 6 8} \pm \\
7.98 \\
(3- \\
27.5)\end{array}$ & $\begin{array}{l}2931.29 \pm \\
2730.60 \\
(44.13- \\
8374)\end{array}$ \\
\hline $\begin{array}{l}\text { MON } \\
\text { DAY }\end{array}$ & 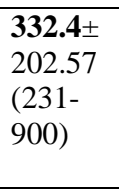 & $\begin{array}{l}\mathbf{5 8 8 0} \pm 6 \\
209.455 \\
(0- \\
16700)\end{array}$ & $\begin{array}{l}\mathbf{6 2 1 2 . 4} \\
\pm \\
6169.69 \\
(231- \\
17021) \\
\end{array}$ & $\begin{array}{l}\mathbf{1 7 . 8} \\
\pm \\
9.22 \\
(1- \\
31) \\
\end{array}$ & $\begin{array}{l}6230.2 \pm \\
6170.83 \\
(249- \\
17035)\end{array}$ & $\begin{array}{l}\mathbf{6 6 . 6 6} \pm \\
35.77 \\
(35.57- \\
160.5)\end{array}$ & $\begin{array}{l}\mathbf{2 9 1 1 .} \\
\mathbf{6 6 6} \pm 2 \\
782.6 \\
96(0- \\
8350) \\
\end{array}$ & $\begin{array}{l}\text { 2978.32 } \\
\pm \\
2807.21 \\
(35.57- \\
8510.5) \\
\end{array}$ & $\begin{array}{l}\mathbf{4 . 0 9} \pm \\
2.315 \\
(.09- \\
7.75)\end{array}$ & $\begin{array}{l}\text { 2982.41 } \pm \\
2808.14 \\
(40.53- \\
8517.5)\end{array}$ \\
\hline $\begin{array}{l}\text { TUES } \\
\text { DAY }\end{array}$ & $\begin{array}{l}\mathbf{5 1 7 . 5 \pm} \\
493.95 \\
(195- \\
1900)\end{array}$ & $\begin{array}{l}\mathbf{5 8 3 0} \pm 6 \\
059.712 \\
(0- \\
16500)\end{array}$ & $\begin{array}{l}\mathbf{6 3 4 7 . 5} \\
\pm \\
5919.40 \\
(341- \\
16867)\end{array}$ & $\begin{array}{l}33.5 \\
\pm \\
8.20 \\
9 \\
(20- \\
46) \\
\end{array}$ & $\begin{array}{l}\mathbf{6 3 8 1} \pm \\
5966.77 \\
(361- \\
16909)\end{array}$ & $\begin{array}{l}\mathbf{9 3 . 8 4} \pm \\
50.25 \\
(48.71- \\
195.5)\end{array}$ & $\begin{array}{l}\mathbf{2 8 6 5} \pm \\
2643 . \\
341 \\
(0- \\
7750)\end{array}$ & $\begin{array}{l}\mathbf{2 9 5 8 . 8 4} \\
\pm \\
2659.96 \\
(48.71- \\
7945.5)\end{array}$ & $\begin{array}{l}\mathbf{7 . 4 8} \pm 3 \\
.97 \\
(2.54- \\
16.5)\end{array}$ & $\begin{array}{l}\text { 2966.32 } \pm \\
2663.24 \\
(51.56- \\
7962)\end{array}$ \\
\hline $\begin{array}{l}\text { WED } \\
\text { NES } \\
\text { DAY }\end{array}$ & $\begin{array}{l}\mathbf{3 6 9 . 5 \pm} \\
262.40( \\
212- \\
1100)\end{array}$ & $\begin{array}{l}\mathbf{5 6 9 2} \pm 5 \\
935.072 \\
(0- \\
16200)\end{array}$ & $\begin{array}{l}\mathbf{6 0 6 1 . 5} \\
\pm \\
5853.40 \\
(262- \\
16500)\end{array}$ & $\begin{array}{l}36.7 \\
\pm \\
63.4 \\
2 \\
(2- \\
216) \\
\end{array}$ & $\begin{array}{l}6098.2 \pm \\
5896.13 \\
(283- \\
16716)\end{array}$ & $\begin{array}{l}\mathbf{7 0 . 8 0} \pm 3 \\
2.69 \\
(37.42- \\
150)\end{array}$ & $\begin{array}{l}\mathbf{2 8 1 2 .} \\
\mathbf{6 6 6} \pm 2 \\
644.9 \\
55(0- \\
8100)\end{array}$ & $\begin{array}{l}\mathbf{2 8 8 3 . 4 7} \\
\pm \\
2661.28 \\
(37.42- \\
8250)\end{array}$ & $\begin{array}{l}\mathbf{1 3 . 5 7 \pm} \\
33.21 \\
(0.5- \\
108)\end{array}$ & $\begin{array}{l}\mathbf{2 8 9 7 . 0 3} \pm \\
2685.76 \\
(40.42- \\
8358)\end{array}$ \\
\hline $\begin{array}{l}\text { THU } \\
\text { RS } \\
\text { DAY } \\
\end{array}$ & $\begin{array}{l}\mathbf{3 1 0 . 3 \pm} \\
107.38 \\
(235- \\
\end{array}$ & $\begin{array}{l}\mathbf{5 8 6 0} \pm 6 \\
255.344 \\
(0-\end{array}$ & $\begin{array}{l}\mathbf{6 1 7 0 . 3} \\
\pm \\
7819.36 \\
\end{array}$ & $\begin{array}{l}\mathbf{2 2 . 4} \\
\pm \\
11.0 \\
\end{array}$ & 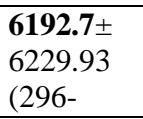 & $\begin{array}{l}\mathbf{6 4 . 6 6 \pm} \\
31.05 \\
(38.57- \\
\end{array}$ & $\begin{array}{l}\mathbf{2 8 8 0 \pm} \\
2740 . \\
965(0 \\
\end{array}$ & $\begin{array}{l}\mathbf{2 9 4 4 . 6 6} \\
\pm \\
2764.07 \\
\end{array}$ & $\begin{array}{l}\mathbf{4 . 4 9 3} \pm \\
2.154 \\
(1.36-\end{array}$ & $\begin{array}{l}\text { 2949.15士 } \\
2764.45( \\
42.284-\end{array}$ \\
\hline $\begin{array}{l}\text { FRID } \\
\text { AY }\end{array}$ & $\begin{array}{l}\text { 411.4 } \pm \\
526.81( \\
150- \\
1900)\end{array}$ & $\begin{array}{l}\mathbf{5 9 3 0} \pm 6 \\
341.758 \\
(0- \\
16900)\end{array}$ & $\begin{array}{l}\mathbf{6 3 4 1 . 4} \\
\pm \\
6185.39 \\
(264- \\
17242) \\
\end{array}$ & $\begin{array}{l}\mathbf{2 4 . 6} \\
\pm \\
6.75 \\
(14- \\
37) \\
\end{array}$ & $\begin{array}{l}\mathbf{6 3 6 6} \pm \\
6188.59 \\
(285- \\
17274)\end{array}$ & $\begin{array}{l}\mathbf{6 5 . 7 3} \pm 3 \\
9.53 \\
(38.57- \\
172.72)\end{array}$ & $\begin{array}{l}\mathbf{2 8 9 3} . \\
\mathbf{3 3 4} \pm 2 \\
753.7 \\
99(0- \\
8400) \\
\end{array}$ & $\begin{array}{l}\mathbf{2 9 5 9 . 0 6} \\
\pm \\
2748.73 \\
(44- \\
8475) \\
\end{array}$ & $\begin{array}{l}\mathbf{5 . 5 1 8} \pm \\
3.290 \\
(1.90- \\
13.5)\end{array}$ & $\begin{array}{l}\mathbf{2 9 6 4 . 5 8} \pm \\
2751.54 \\
(41.99- \\
8488.5)\end{array}$ \\
\hline $\begin{array}{l}\text { SAT } \\
\text { UR } \\
\text { DAY }\end{array}$ & $\begin{array}{l}\mathbf{3 1 9 . 3 \pm} \\
145.65 \\
(210- \\
717)\end{array}$ & $\begin{array}{l}\mathbf{5 8 9 5} \pm 6 \\
279.705 \\
(0- \\
16700)\end{array}$ & $\begin{array}{l}\mathbf{6 2 1 4 . 3} \\
\pm \\
6245.26 \\
(254- \\
17000)\end{array}$ & $\begin{array}{l}\mathbf{1 7 . 2} \\
\mathbf{5 \pm} \\
14.5 \\
9 \\
(2.5- \\
54) \\
\end{array}$ & $\begin{array}{l}\mathbf{6 2 3 1 . 5 5} \pm \\
6250.60 \\
(271- \\
17054)\end{array}$ & $\begin{array}{l}\mathbf{6 4 . 7 5} \pm \\
29.96 \\
(36.28- \\
145.5)\end{array}$ & $\begin{array}{l}\mathbf{2 9 1 4 .} \\
\mathbf{1 6} \pm 27 \\
87.96 \\
5 \quad(0- \\
8250)\end{array}$ & $\begin{array}{l}\text { 2978.92 } \\
\pm \\
2809.27 \\
(36.28- \\
8395.5)\end{array}$ & $\begin{array}{l}\mathbf{3 . 3 5} \pm \\
2.950 \\
(1.25- \\
10.8)\end{array}$ & $\begin{array}{l}\text { 2982.271 } \\
\pm \\
2810.26 \\
(38.7- \\
8396.75)\end{array}$ \\
\hline $\begin{array}{l}\text { Avg. } \\
\text { /day }\end{array}$ & $\begin{array}{l}\mathbf{3 6 8 . 4 8} \\
\mathbf{\pm} \\
\mathbf{7 4 . 8 3} \\
(310.3- \\
517.5)\end{array}$ & $\begin{array}{l}\mathbf{5 8 2 9 . 7 1} \\
\mathbf{4} \pm \mathbf{8 9 . 9 5} \\
\mathbf{3 1 6} \\
(5692- \\
5930)\end{array}$ & $\begin{array}{l}\mathbf{6 1 9 8 . 2} \\
\mathbf{+ 1 2 0 . 9} \\
\mathbf{3 3 9} \\
(6040- \\
6347.5)\end{array}$ & $\begin{array}{l}\mathbf{3 2 . 3} \\
\mathbf{7 \pm} \\
\mathbf{1 9 . 9} \\
\mathbf{4} \\
(17.8 \\
- \\
74.4)\end{array}$ & $\begin{array}{l}\text { 6230.57士 } \\
\mathbf{1 1 0 . 6 2 1 7} \\
(6098.2- \\
6381)\end{array}$ & $\begin{array}{l}\mathbf{7 0 . 3 6} \pm \mathbf{1} \\
\mathbf{0 . 5 5} \\
(64.66- \\
93.84)\end{array}$ & $\begin{array}{l}2875 . \\
\mathbf{3 3 2} \pm 3 \\
\mathbf{6 . 1 1 1} \\
\mathbf{0 4} \\
(2812 . \\
66- \\
2914 . \\
16) \\
\end{array}$ & $\begin{array}{l}\mathbf{2 9 4 5 . 6 9} \\
\pm \\
\mathbf{3 4 . 7 3} \\
(2883.47 \\
- \\
2978.92)\end{array}$ & $\begin{array}{l}\mathbf{7 . 5 9 \pm} \\
\mathbf{4 . 6 5} \\
(3.35- \\
14.68)\end{array}$ & 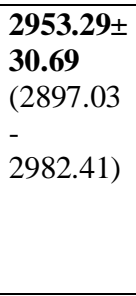 \\
\hline
\end{tabular}


Solid waste generation per day in high, middle and low income in households of Parwah village(Table IV)

\begin{tabular}{|l|l|l|l|}
\hline $\begin{array}{l}\text { Type of } \\
\text { household }\end{array}$ & Biodegradable waste (gm) & $\begin{array}{l}\text { Non-biodegradable waste } \\
(\mathrm{gm})\end{array}$ & TOTAL (gm) \\
\hline High & $8496.75 \pm$ & $45.12 \pm$ & $8541.88 \pm$ \\
& 1519.48 & 21.10 & 1525.439 \\
& $(5092.6-9313.2)$ & $(20.8-76.6)$ & $(5120.6-9363.4-)$ \\
\hline Middle & $4879.15 \pm$ & $33.00 \pm$ & \\
& 373.03 & 10.41 & $4912.16 \pm$ \\
& $(4118.9-5219.1)$ & $(21.3-52.1)$ & $377.1041(4146.1-5250.5)$ \\
\hline Low & $6198.2 \pm$ & $32.37 \pm$ & $6230.57 \pm$ \\
& 120.9339 & 19.94 & 110.6217 \\
& $(6040-6347.5)$ & $(17.8-74.4)$ & $(6098.2-6381)$ \\
\hline Avg. solid & $\mathrm{X}=$ & $\mathrm{Y}=$ & $\mathrm{Z}=$ \\
Waste/average & $6524.7 \pm 1830.767$ & $36.83 \pm 7.186258$ & $6561.54 \pm 1837.355$ \\
house/day & & & \\
\hline
\end{tabular}

Total average solid waste generation in the study area $(w)=Z \times$ No. of households in the study area

$=6561.54 \times 250=1640385 \mathrm{gms}(1640.38 \mathrm{~kg})$

Total solid waste generation $/$ week $(1640.38 \mathrm{~kg} \times 7)=11482.66 \mathrm{~kg}$

Total solid waste generation/month $(1640.38 \mathrm{~kg} \times 30)=49211.4 \mathrm{~kg}$

Total solid waste generation/year $(1640.38 \mathrm{~kg} \times 365) \quad=598738.7 \mathrm{~kg}$

Table XXX Average per week, per month and per year solid waste generation (Table V)

\begin{tabular}{|l|l|l|l|l|}
\hline Type of waste & $\begin{array}{l}\text { Avg. solid } \\
\text { waste/day }\end{array}$ & Avg. Solid waste /week & $\begin{array}{l}\text { Avg. solid } \\
\text { waste/month }\end{array}$ & $\begin{array}{l}\text { Avg. } \\
\text { waste/year }\end{array}$ \\
\hline Biodegradable & $1631.17 \mathrm{~kg}$ & $111418.19 \mathrm{~kg}$ & $48935.1 \mathrm{~kg}$ & $595377.05 \mathrm{~kg}$ \\
\hline $\begin{array}{l}\text { Non- } \\
\text { biodegradable }\end{array}$ & $9.20 \mathrm{~kg}$ & $64.4 \mathrm{~kg}$ & $276 \mathrm{~kg}$ & \\
\hline Total solid waste & $1640.38 \mathrm{~kg}$ & $11482.66 \mathrm{~kg}$ & $49211.4 \mathrm{~kg}$ & $3358 \mathrm{~kg}$ \\
\hline
\end{tabular}

\section{Conclusion}

The study concludes that wealthier households generally generate more waste. compilation of the data of all the households of the study area reveal that the average solid waste generation in average household of the study area was observed to be $6561.543 \pm 1837.35 \mathrm{~g} /$ house/day i.e. $6.561 \pm 1.837 \mathrm{~kg} / \mathrm{house} / \mathrm{day}$. The generation of solid waste was observed to be positively correlated with family size in all types of households i.e. high income households $(r=0.77, p<0.05)$, middle income households $(r=0.59, p<0.05)$ and low income households $(\mathrm{r}=0.1, \mathrm{p}<0.05)$. Overall, the data presented here could be valuable for organizing waste management in KMC and the methods used to derive the data can be useful procedure in examining waste stream characteristics in other developing countries.

\section{References}

[1]. Qdais, H.A.A., M.F.Hamoda, J.Newham (1997) Analysis of Residential Solid Waste At Generation Sites. Waste Manag Res. 15(4):395-405.

[2]. Ansari, M. S. Al. (2012) International journal of water resources and environmental engineering, 4 (5): $152-161$.

[3]. Chakarabarti, T. (1993) Legal aspects of hazardous waste management in India. Journal of Ind. Assoc. of Environ. Management, 20(1-3):1-5.

[4]. Gamse, T. and C. Medina (2010) Development of Solid Waste Management practices in Indonesia-European journal of scientific research, 40(2):199-210.

[5]. Mukherjee, A.B and P.Roy (1989) Calcutta metropolitan waste, its characteristics, Pollution load and disposal problem. Env. Eco. 7 (4):1019-1022.

[6]. Pasrija, H.B.D., S. Chander and S. Kumar (1995) Solid waste management in Chandigarh city. In: Environmental pollution and protection (Ed. M.R Garg V.K. Bansal, N.S. Tiwana Deep and Deep Publications, New Delhi.

[7]. Rao, J. K. and M.V. Shantaram (1996 a) Solid pollution due to the disposal of solid waste at landfill site at Hyderabad. Ind. J. Env. Prot., 16(5):373-385.

[8]. Rao, J. K. and M.V. Shantaram (1996 b) heavy metals and micro nutrient contents and their relative availability in stabilized urban solid waste of Hyderabad. Poll. Res., 15(3)271-272.

[9]. Sharholy, M., K. Ahmad, G. Mahmood, and R.C. Trivedi (2007) Municipal Solid Waste Management in Indian cities. International Journal of Integrated Waste Management, 28(2):459-467.

[10]. Sinha, R.K. and R. Rawat (1991) Waste recycling and reutilization essential for environment safety and sustainable development-a case study. J. Eco. Bio., 3(3):193-195.

[11]. Sujauddin,M.,S.M.S.Huda,A.T.M.R.Hoque (2008) Household solid waste characteristics and management in Chittagong, Bangladesh. Elsevier waste management., 28(9):1688-1695. 